\title{
On modeling mixed hardening in computational plasticity
}

\author{
Klaus-Jürgen Bathe ${ }^{\text {a,* }}$, Francisco Javier Montáns ${ }^{b}$ \\ a Department of Mechanical Engineering, Massachusetts Institute of Technology, 77 Massachusetts Avenue, Room 3-356, \\ Cambridge, MA 02139, USA \\ ${ }^{\mathrm{b}}$ Escuela Técnica Superior de Ingenieros Industriales, Universidad de Castilla-La Mancha, 13071 Ciudad Real, Spain
}

Received 14 April 2003; accepted 12 August 2003

\begin{abstract}
We address herein the calculation of Prager's hardening parameter in computational plasticity when mixed hardening is considered. We consider two approaches to evaluate the mixed hardening response; namely, based on splitting the plastic strains and based on splitting the plastic modulus. For a one-dimensional stress-strain curve with nonlinear hardening, the proper calculation of Prager's hardening parameter is demonstrated and some comparisons and insight are provided.
\end{abstract}

(c) 2003 Elsevier Ltd. All rights reserved.

Keywords: Computational plasticity; Cyclic response; Prager's rule; Mixed hardening

\section{Introduction}

The development of material models and their use in computational plasticity is of much importance and interest [1,2]. Numerous papers have been written on the use of classical and improved inelastic material models, and many new models to capture the nonlinear behavior of materials have been proposed.

Frequently, with these models, the hardening and cyclic behavior shall be captured, and this is in many cases accomplished using Prager's mixed hardening approach [3]. Prager's hardening parameter is computed using one-dimensional conditions and then applied to multi-dimensional cases, see, for example, [4,5]. However, while Prager's theory is clear, it must be applied judiciously in computational plasticity. We consider in this paper the case of nonlinear mixed hardening and discuss and compare two approaches - one based on splitting the plastic strains and one based on splitting the plastic modulus - to obtain a value for Prager's harden-

\footnotetext{
${ }^{*}$ Corresponding author. Tel.: +1-617-253-6645; fax: +1-617253-2275.

E-mail address: kjb@mit.edu (K.J. Bathe).
}

ing parameter. In the case of splitting the plastic strains, the result is different from the parameter frequently used in the literature, see for example [6-10]. However, with the result given in this paper we reproduce the onedimensional test results in a physical manner, whereas using the parameter frequently given in the literature, a response inconsistent with one-dimensional test results is obtained. In the case of splitting the plastic modulus, the one-dimensional test result is also reproduced exactly but the procedure may be regarded to be less physical.

We do not comment on inelastic material models, and do not wish to discuss the advantages and disadvantages of the assumptions of Prager's model. Indeed many other and more comprehensive models to represent hardening characteristics have been proposed. However, the simple approach of Prager is still widely used in engineering practice and for this reason we pursued this study.

Hence, the only objective of this paper is to focus on the proper evaluation of Prager's hardening parameter corresponding to a one-dimensional mathematical model of a generic physical experiment. This evaluation shall clear up some misconceptions and of course give some insight into the modeling of mixed hardening in computational plasticity. 


\section{Derivation of hardening parameters}

Let us consider the general nonlinear elasto-plastic response corresponding to a specimen in one-dimensional tensile stress conditions, as shown in Fig. 1. The response in the figure corresponds to a mathematical model of plasticity and is assumed to closely (and perhaps exactly) represent the measured data obtained in a tensile test. This response must clearly be reproduced by any computational scheme and in any conditions of assumed hardening, that is, in kinematic, isotropic, or mixed hardening - as long as we subject the specimen only to monotonically increasing tensile strain conditions. As is usually done, we use this one-dimensional response to evaluate Prager's mixed hardening parameter. This result, with of course also uniaxial cyclic response data, is then employed in general multidimensional stress analysis.

To proceed with the evaluation of Prager's mixed hardening parameter, we recall some classical governing relations corresponding to von Mises plasticity, but only those that we shall actually use in our derivations.

For the next developments, consider the following decomposition of the plastic strain increments

$\mathrm{d} \boldsymbol{e}^{\mathrm{p}}=\mathrm{d} \boldsymbol{e}^{\mathrm{pi}}+\mathrm{d} \boldsymbol{e}^{\mathrm{pk}}=M \mathrm{~d} \boldsymbol{e}^{\mathrm{p}}+(1-M) \mathrm{d} \boldsymbol{e}^{\mathrm{p}}$

where $\mathrm{d} \boldsymbol{e}^{\mathrm{pi}}$ and $\mathrm{d} \boldsymbol{e}^{\mathrm{pk}}$ are, respectively, the isotropic and kinematic contributions. We note that these strains are hence assumed to be co-linear. The hardening conditions correspond to isotropic hardening, $M=1$, kinematic hardening, $M=0$, or general mixed hardening, $0<M<$ 1. We are interested in this last case.

The yield function assuming mixed hardening is

$f_{y}:=\frac{1}{2} \hat{\boldsymbol{s}}: \hat{\boldsymbol{s}}-\frac{1}{3} \sigma_{y}^{2}$

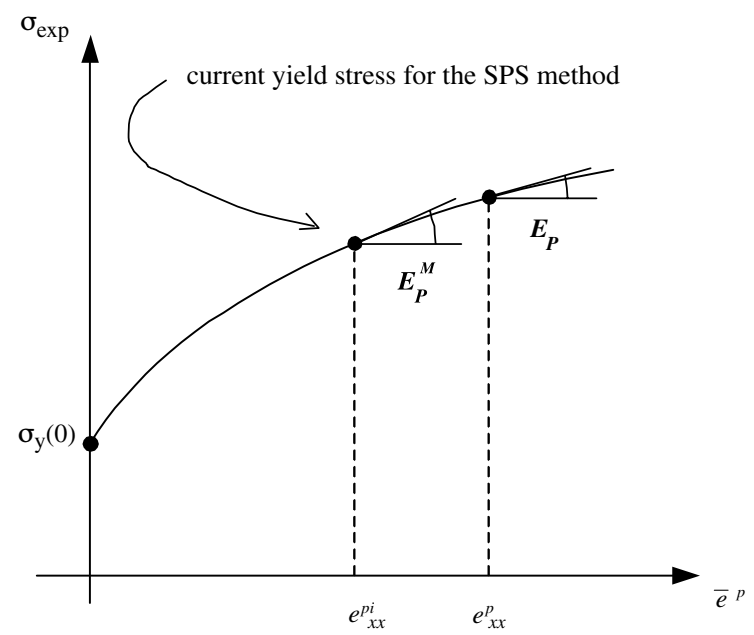

Fig. 1. One-dimensional monotonically increasing tensile stress situation (yield curve). A generic yield curve. where $\hat{\boldsymbol{s}}:=\boldsymbol{s}-\boldsymbol{\alpha}$ are the deviatoric stresses $\boldsymbol{s}:=\operatorname{dev}(\boldsymbol{\sigma})$ shifted by the back stresses $\boldsymbol{\alpha}$ and $\sigma_{y}$ is the effective yield stress, and of course during yielding $f_{y}=0$.

There are two main approaches that are used to obtain $\sigma_{y}$ and $\boldsymbol{\alpha}$. In the first approach, see for example [9], which we call the "splitting of plastic strains (SPS) method", we consider $e^{\mathrm{pi}}$ and $\boldsymbol{e}^{\mathrm{pk}}$ as main variables (computing $\boldsymbol{e}^{\mathrm{p}}$ via Eq. (1)) and $\sigma_{y}$ as a function of the effective isotropic plastic strain $\sigma_{y}=\tilde{\sigma}_{y}\left(\bar{e}^{\mathrm{pi}}\right)$. In the second approach, see for example [11], which we call the "splitting of plastic modulus (SPM) method", we consider the total effective plastic strain $\bar{e}^{\mathrm{p}}$ as the main variable and $\sigma_{y}$ as a function of that strain, $\sigma_{y}=\hat{\sigma}_{y}\left(\bar{e}^{\mathrm{p}}\right)$.

Given a variation of the effective plastic strain $\mathrm{d} \bar{e}^{\mathrm{p}}$, the variation of $\sigma_{y}$ is in the SPS method

$\mathrm{d} \sigma_{y} \equiv \delta \tilde{\sigma}_{y}=\frac{\mathrm{d} \tilde{\sigma}_{y}}{\mathrm{~d} \bar{e}^{\mathrm{pi}}} \delta \bar{e}^{\mathrm{pi}}=\frac{\mathrm{d} \tilde{\sigma}_{y}}{\mathrm{~d} \bar{e}^{\mathrm{pi}}} \frac{\partial \bar{e}^{\mathrm{pi}}}{\partial \bar{e}^{\mathrm{p}}} \mathrm{d} \bar{e}^{\mathrm{p}}:=E_{\mathrm{p}}^{M} M \mathrm{~d} \bar{e}^{\mathrm{p}}$

and in the SPM method

$\mathrm{d} \sigma_{y} \equiv \mathrm{d} \hat{\sigma}_{y}=\frac{\mathrm{d} \hat{\sigma}_{y}}{\mathrm{~d} \bar{e}^{\mathrm{p}}} \mathrm{d} \bar{e}^{\mathrm{p}}:=K^{\prime} \mathrm{d} \bar{e}^{\mathrm{p}}$

In Eq. (3a) we use Eq. (1) and thus have $\partial \bar{e}^{\mathrm{pi}} / \partial \bar{e}^{\mathrm{p}}=M$. We also employ $\delta(\cdot)$ to emphasize that it is the variation due to $\mathrm{d} \bar{e}^{\mathrm{p}}$ and not due to $\mathrm{d} \bar{e}^{\mathrm{pi}}$ (i.e. $\delta \tilde{\sigma}_{y}=M \mathrm{~d} \tilde{\sigma}_{y}$ where $\mathrm{d} \tilde{\sigma}_{y}$ is the variation in the yield stress at $\bar{e}^{\mathrm{pi}}$ corresponding to $\left.\mathrm{d} \bar{e}^{\mathrm{p}}\right)$. We note that, in Eq. (2), consistent with the SPS method, the value of $\sigma_{y}$ is evaluated at $\bar{e}^{\mathrm{pi}}$, whereas consistent with the SPM method, the value of $\sigma_{y}$ is evaluated at $\bar{e}^{\mathrm{p}}$.

Prager's hardening rule assumes that the evolution of the back stresses $\alpha$ is proportional to that of the plastic strains $\boldsymbol{e}^{\mathrm{p}}$. Again, both approaches are applicable, i.e., $\mathrm{d} \alpha$ may be considered as a function of the kinematic plastic strain increments $\mathrm{d} \boldsymbol{\alpha}=\delta \tilde{\boldsymbol{\alpha}}\left(\delta \boldsymbol{e}^{\mathrm{pk}}\right)$ or as a function of the total plastic strain increments $\mathrm{d} \boldsymbol{\alpha}=\mathrm{d} \hat{\boldsymbol{\alpha}}\left(\mathrm{d} \boldsymbol{e}^{\mathrm{p}}\right)$.

For the evaluation of Prager's hardening parameter, we consider uniaxial conditions corresponding to the $x$ axis. Then given a variation $\mathrm{d} e_{x x}^{\mathrm{p}}$ we obtain for the SPS method,

$\mathrm{d} \alpha_{x x} \equiv \delta \tilde{\alpha}_{x x}=\frac{\mathrm{d} \tilde{\alpha}_{x x}}{\mathrm{~d} e_{x x}^{\mathrm{pk}}} \frac{\partial e_{x x}^{\mathrm{pk}}}{\partial e_{x x}^{\mathrm{p}}} \mathrm{d} e_{x x}^{\mathrm{p}}:=C_{\mathrm{p}}(1-M) \mathrm{d} e_{x x}^{\mathrm{p}}$

and for the SPM method

$\mathrm{d} \alpha_{x x} \equiv \mathrm{d} \hat{\alpha}_{x x}=\frac{\mathrm{d} \hat{\alpha}_{x x}}{\mathrm{~d} e_{x x}^{\mathrm{p}}} \mathrm{d} e_{x x}^{\mathrm{p}}:=H^{\prime} \mathrm{d} e_{x x}^{\mathrm{p}}$

Of course in the uniaxial loading considered

$\mathrm{d} \sigma_{x x}=\frac{\partial \sigma_{x x}}{\partial e_{x x}^{\mathrm{p}}} \mathrm{d} e_{x x}^{\mathrm{p}}:=E_{\mathrm{p}} \mathrm{d} e_{x x}^{\mathrm{p}}$

where $E_{\mathrm{p}}$ is the tangent of the (experimental, prescribed) uniaxial stress-plastic strain curve. Using Eq. (2) for the uniaxial condition and performing the variation on $f_{y}$ 
(which must be zero by the consistency requirement) we obtain

$\mathrm{d} \sigma_{x x}-\frac{3}{2} \mathrm{~d} \alpha_{x x}-\mathrm{d} \sigma_{y}=0$

For the general case we have $\mathrm{d} e_{x x}^{\mathrm{p}} \neq 0$. Hence, substituting Eqs. (3a), (4a) and (5) for the SPS method we obtain

$E_{\mathrm{p}}-\frac{3}{2} C_{\mathrm{p}}(1-M)-E_{\mathrm{p}}^{M} M=0$

and using Eqs. (3b), (4b) and (5) for the SPM method we obtain

$E_{\mathrm{p}}-\frac{3}{2} H^{\prime}-K^{\prime}=0$

Eq. (7a) is trivial when $M=1$ (simply saying that $E_{\mathrm{p}}^{M}=E_{\mathrm{p}}$ since $\left.\mathrm{d} \bar{e}^{\mathrm{pi}}=\mathrm{d} \bar{e}^{\mathrm{p}}\right)$, but otherwise gives $C_{\mathrm{p}}$ as

$C_{\mathrm{p}}=\frac{2}{3} \frac{E_{\mathrm{p}}-M E_{\mathrm{p}}^{M}}{1-M}$

where from Eq. (3a) $E_{\mathrm{p}}^{M}=\left.E_{\mathrm{p}}\right|_{\bar{e}^{\mathrm{p}}}$. Here we emphasize that consistent with this approach $E_{\mathrm{p}}^{M}$ is the tangent of the stress-plastic strain curve at the effective isotropic plastic strain.

The result given in Eq. (8) is different from the result given by other authors, who simply use for this approach

$C_{\mathrm{p}}=\frac{2}{3} E_{\mathrm{p}}$

see for example [6-10]. Here we should note that Crisfield in Ref. [9] obtained Eq. (7a) but then erroneously concluded that Eq. (9) must hold. The value given in Eq. (9) holds for the case of kinematic hardening, that is when $M=0$, and for the case when a bilinear stressstrain curve is assumed, that is, when $E_{\mathrm{p}}^{M}=\left.E_{\mathrm{p}}\right|_{e^{\mathrm{pi}}}=$ $\left.E_{\mathrm{p}}\right|_{\bar{e}^{\mathrm{p}}}$. But in general nonlinear mixed hardening conditions, the formula in Eq. (8) should be used.

If the value given in Eq. (8) is used, the one-dimensional response given in Fig. 1 is exactly reproduced, and for any value of $M$. On the other hand, if the value in Eq. (9) is used, the model response of Fig. 1 is not exactly reproduced. The discrepancy clearly depends on the nonlinearity of the yield curve and the value of $M$. However, in computational plasticity it is obviously imperative to reproduce the one-dimensional response exactly, which merely corresponds to saying that the "response output must be equal to the response input".

Fig. 2 shows an example of a yield curve and the results obtained using Eq. (8) (the results are equal to the yield curve) and the results obtained using Eq. (9). Here the case of $M=0.5$ is considered and a considerable discrepancy is seen between the predicted response using Eq. (9) and the response that should be obtained. It is

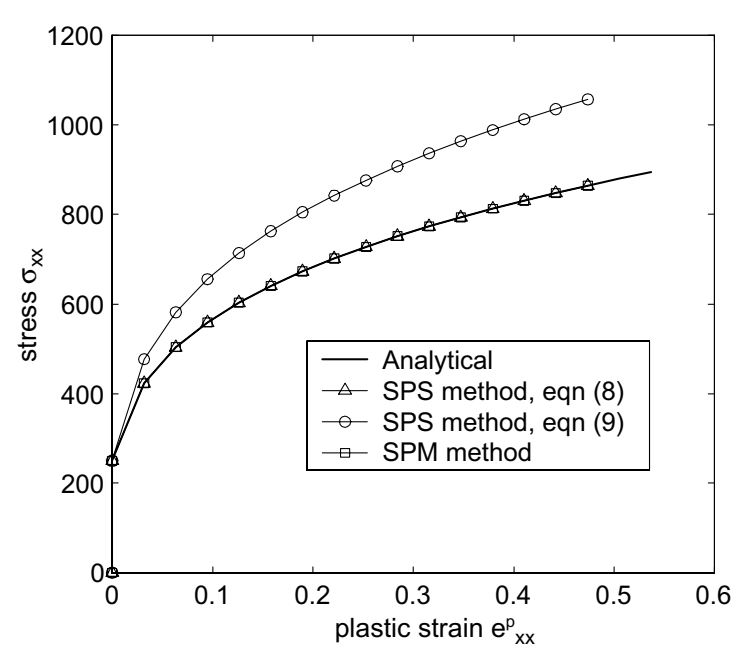

Fig. 2. Stress-strain curves; Ramberg-Osgood model (the numerical error in the integration is negligible).

clear that it is important in practice to use Eq. (8) in the computations.

In the SPM method, the condition (7b) must hold. In this case authors typically select $K=\widehat{M} \sigma_{\text {exp }}$ and $H=$ $\frac{2}{3}(1-\widehat{M}) \sigma_{\exp }$ such that

$K^{\prime}=\widehat{M} E_{\mathrm{p}}$

$H^{\prime}=\frac{2}{3}(1-\widehat{M}) E_{\mathrm{p}}$

where $\widehat{M}$ is interpreted to have a similar meaning as $M$ and $\sigma_{\exp }\left(e_{x x}^{\mathrm{p}}\right)$ is the experimental (or analytical) stressstrain curve [11-14].

We should note that except for bilinear stress-strain curves, both approaches differ in the modeling of mixed hardening

- In general, $\widehat{M} E_{\mathrm{p}} \neq M E_{\mathrm{p}}^{M}$ since $E_{\mathrm{p}}^{M}$ is evaluated at $e^{\mathrm{pi}}$ whereas $E_{\mathrm{p}}$ is evaluated at $\bar{e}^{\mathrm{p}}$.

- In general, $C_{\mathrm{p}}(1-M) \neq H^{\prime}$, since $C_{\mathrm{p}}$ must be obtained via Eq. (8) while $H^{\prime}$ is prescribed as given in Eq. (10b).

- In the SPS method the yield stress in Eq. (2) is $\tilde{\sigma}_{y}$, a function of $\bar{e}^{\mathrm{pi}}$ and, therefore, evaluated at $\bar{e}^{\mathrm{pi}}$. In the SPM method the yield stress is $\hat{\sigma}_{y}$, a function of $\bar{e}^{\mathrm{p}}$ and evaluated at $\bar{e}^{\mathrm{p}}$.

- In essence, the SPS method makes use of the assumption in Eq. (1) whereas the SPM method makes use of the assumption in Eqs. (10a) and (10b).

Obviously, the same monotonic uniaxial stress-strain curve is obtained in both approaches provided Eq. (8) is used in the SPS method (of course Eq. (7b) is automatically satisfied in the SPM method by using Eqs. (10a) and (10b)), but the cyclic behavior for the case of 


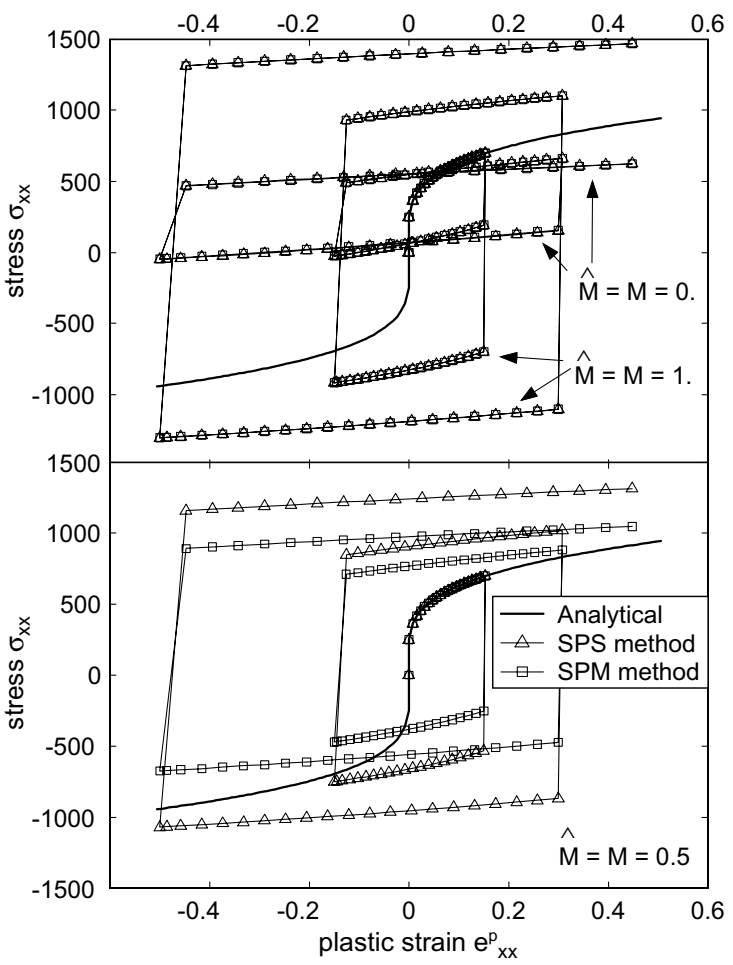

Fig. 3. Predictions under cyclic loading for different values of $M, \widehat{M}$. The SPS and SPM methods yield the same response when $M=\widehat{M}=0$ and $M=\widehat{M}=1$.

mixed hardening is in general clearly different, as shown for example in Fig. 3. Both approaches are equally valid since from a uniaxial experimental monotonic curve nothing can be said about which amount corresponds to each type of hardening.

For a more accurate modeling in cyclic loading, a variable mixed hardening such as, for example in the SPS method

$M=M_{\infty}+\left(M_{0}-M_{\infty}\right) \exp \left(-\eta \bar{e}^{\mathrm{p}}\right)$

and in the SPM method

$\widehat{M}=\widehat{M}_{\infty}+\left(\widehat{M}_{0}-\widehat{M}_{\infty}\right) \exp \left(-\eta \bar{e}^{\mathrm{p}}\right)$

may yield better results for the prediction of the Bauschinger effect at different strain levels. An illustrative example comparing the response predicted using the SPS and SPM methods, but without comparison with experimental results, is given in Fig. 4.

Note that in Figs. 1-4, the strain levels reached are rather large merely for demonstration purposes. In practice, a large strain formulation would need to be used [13-15].

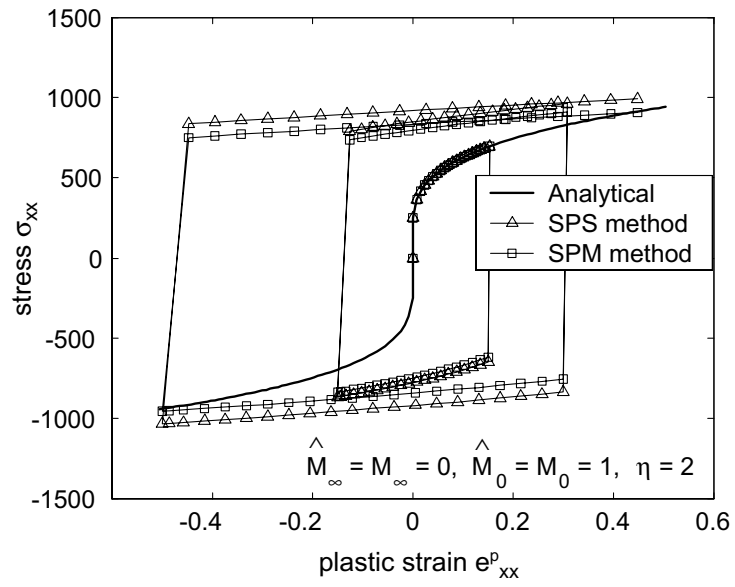

Fig. 4. Cyclic response prediction using the variables $M, \widehat{M}$ as given by Eqs. (11a) and (11b).

\section{Work-conjugacy - the principle of maximum plastic dissipation}

In each approach, the dissipation function may be written in terms of the main variables considered. We have for the SPS method

$\mathrm{d} \widetilde{\mathscr{D}}^{\mathrm{p}}=\boldsymbol{\sigma}: \mathrm{d} \boldsymbol{e}^{\mathrm{p}}+\tilde{\boldsymbol{\alpha}}: \mathrm{d} \tilde{\xi}+\tilde{\sigma}_{y} \mathrm{~d} \tilde{\zeta}$

and for the SPM method

$\mathrm{d} \widehat{\mathscr{D}}^{\mathrm{p}}=\boldsymbol{\sigma}: \mathrm{d} \boldsymbol{e}^{\mathrm{p}}+\hat{\boldsymbol{\alpha}}: \mathrm{d} \hat{\boldsymbol{\xi}}+\hat{\sigma}_{y} \mathrm{~d} \hat{\zeta}$

where $\tilde{\xi}, \tilde{\varsigma}, \hat{\xi}$ and $\hat{\varsigma}$. are internal variables work-conjugate to $\tilde{\boldsymbol{\alpha}}, \tilde{\sigma}_{y}, \hat{\boldsymbol{\alpha}}$ and $\hat{\sigma}_{y}$ respectively. Using a threedimensional version of Eq. (6) we have

$\mathrm{d} F_{y} \equiv \boldsymbol{n}:(\mathrm{d} \boldsymbol{s}-\mathrm{d} \boldsymbol{\alpha})-\sqrt{\frac{2}{3}} \mathrm{~d} \sigma_{y}=0$

where $\boldsymbol{n}:=(\boldsymbol{s}-\boldsymbol{\alpha}) /\|\boldsymbol{s}-\boldsymbol{\alpha}\|$. For the SPS method this equation may be written as

$\mathrm{d} \widetilde{F}_{y} \equiv \boldsymbol{n}:(\mathrm{d} \boldsymbol{s}-\delta \tilde{\boldsymbol{\alpha}})-\sqrt{\frac{2}{3}} \delta \tilde{\sigma}_{y}=0$

whereas for the SPM method we have

$\mathrm{d} \widehat{F}_{y} \equiv \boldsymbol{n}:(\mathrm{d} \boldsymbol{s}-\mathrm{d} \hat{\boldsymbol{\alpha}})-\sqrt{\frac{2}{3}} \mathrm{~d} \hat{\sigma}_{y}=0$

If we then employ Eqs. (3a) and (4a) to write the expression in Eq. (14a) as a function of the variations with respect to their respective primary functions (i.e. $\delta \tilde{\sigma}_{y}=M \mathrm{~d} \tilde{\sigma}_{y}$ and $\left.\delta \tilde{\boldsymbol{\alpha}}=(1-M) \mathrm{d} \tilde{\boldsymbol{\alpha}}\right)$ we obtain

$\mathrm{d} \widetilde{F}_{y} \equiv \boldsymbol{n}:[\mathrm{d} \boldsymbol{s}-(1-M) \mathrm{d} \tilde{\boldsymbol{\alpha}}]-\sqrt{\frac{2}{3}} M \mathrm{~d} \tilde{\sigma}_{y}=0$

The Lagrangians of the constrained principle for the SPS and SPM methods are, respectively, $\widetilde{L}=\mathrm{d} \widetilde{\mathscr{D}}^{\mathrm{p}}$ $\mathrm{d} \tilde{\lambda} \widetilde{F}_{y}$ and $\widehat{L}=\mathrm{d} \widehat{\mathscr{D}}^{\mathrm{p}}-\mathrm{d} \hat{\lambda} \widehat{F}_{y}$. The principle of maximum plastic dissipation yields in the SPS method 
$\nabla \widetilde{L}=\mathbf{0} \Rightarrow\left\{\begin{array}{l}\frac{\partial \tilde{L}}{\partial \boldsymbol{\sigma}}=0 \Rightarrow \mathrm{d} \boldsymbol{e}^{\mathrm{p}}=\mathrm{d} \tilde{\lambda} \boldsymbol{n} \\ \frac{\partial \tilde{L}}{\partial \tilde{\alpha}}=0 \Rightarrow \mathrm{d} \tilde{\xi}=-\mathrm{d} \tilde{\lambda}(1-M) \boldsymbol{n}=-\mathrm{d} \boldsymbol{e}^{\mathrm{pk}} \\ \frac{\partial \tilde{L}}{\partial \tilde{\sigma}_{y}}=0 \Rightarrow \mathrm{d} \tilde{\zeta}=-\mathrm{d} \tilde{\lambda} M \sqrt{\frac{2}{3}}=-\mathrm{d} \bar{e}^{\mathrm{pi}}\end{array}\right.$

and in the SPM method

$\nabla \widehat{L}=\mathbf{0} \Rightarrow\left\{\begin{array}{l}\frac{\partial \hat{L}}{\partial \boldsymbol{\sigma}}=0 \Rightarrow \mathrm{d} \boldsymbol{e}^{\mathrm{p}}=\mathrm{d} \hat{\lambda} \boldsymbol{n} \\ \frac{\partial \hat{L}}{\partial \hat{\alpha}}=0 \Rightarrow \mathrm{d} \hat{\xi}=-\mathrm{d} \hat{\lambda} \boldsymbol{n}=-\mathrm{d} \boldsymbol{e}^{\mathrm{p}} \\ \frac{\partial \hat{L}}{\partial \hat{\sigma}_{y}}=0 \Rightarrow \mathrm{d} \hat{\zeta}=-\mathrm{d} \hat{\lambda} \sqrt{\frac{2}{3}}=-\mathrm{d} \bar{e}^{\mathrm{p}}\end{array}\right.$

These relations show the expected values of the workconjugate variables of $\tilde{\boldsymbol{\alpha}}, \hat{\alpha}, \tilde{\sigma}_{y}$ and $\hat{\sigma}_{y}$ for the SPS and SPM methods.

\section{Concluding remarks}

Mixed hardening in computational plasticity is frequently modeled using Prager's hardening rule. If nonlinear hardening is considered, care must be exercised to employ the rule such that, for all values of $M$, the monotonically increasing one-dimensional stress response is properly captured. We summarized and compared in this paper two approaches that can be used. The first approach is based on splitting the plastic strains (the SPS method) and the second one is based on splitting the plastic modulus (the SPM method). Either approach can be employed but in the SPS method the appropriate formula to evaluate Prager's hardening modulus must be used (and was frequently not employed in the literature).

While we focused here on the case of isotropic timeindependent plasticity, the same concepts and the formula in Eq. (8) are of course also applicable in timedependent conditions and, in the appropriate form, in orthotropic inelastic analysis.

\section{References}

[1] Bathe KJ, editor. Computational fluid and solid mechanics. Elsevier Science; 2001.

[2] Bathe KJ, editor. Computational fluid and solid mechanics 2003. Elsevier Science; 2003.

[3] Prager W. A new method of analyzing stresses and strains in work-hardening plastic solids. J Appl Mech, ASME 1956;23:493-6.

[4] Lemaitre J, Chaboche J-L. Mechanics of solid materials. Cambridge University Press; 1990.

[5] Khan AS, Huang S. Continuum theory of plasticity. John Wiley \& Sons; 1995.

[6] Axelsson K, Samuelsson A. Finite element analysis of elastic-plastic materials displaying mixed hardening. Int $\mathbf{J}$ Numer Meth Eng 1979;14:211-25.

[7] Kojic M. The governing parameter method for implicit integration of viscoplastic constitutive relations for isotropic and orthotropic metals. Comput Mech 1996;19:49-57.

[8] Kojic M, Grujovic N, Slavkovic R, Zivkovic M. A general orthotropic von Mises plasticity model with mixed hardening: model definition and implicit stress integration. Trans ASME 1996;63:376-82.

[9] Crisfield M. Non-linear finite element analysis of solids and structures. Vol. 2. Advanced topics, J Wiley; 1997.

[10] Su M, Gu Q, Guo B. Finite element analysis of steel members under cyclic loading. Finite Elem Anal Des 2002;39:43-54.

[11] Krieg RD, Key SW. Implementation of a time independent plasticity theory into structural computer programs. In: Stricklin JA, Saczalski KJ, editors. Constitutive equations in viscoplasticity: computational and engineering aspects. AMD-20. ASME; 1976. p. 125-37.

[12] Simo JC, Taylor RL. Consistent tangent operators for rate-independent elastoplasticity. Comput Meth Appl Mech Eng 1985;48:101-18.

[13] Eterović AL, Bathe KJ. A hyperelastic-based large strain elasto-plastic constitutive formulation with combined isotropic-kinematic hardening using logarithmic stress and strain measures. Int J Numer Meth Eng 1990;37:1099-114.

[14] Simo JC, Hughes TJR. Computational inelasticity. Springer; 1998.

[15] Bathe KJ. Finite element procedures. Prentice Hall; 1996. 